\title{
Visualização imersiva e colaborativa de moléculas utilizando tecnologia de jogos
}

\author{
Luiz Soares dos Santos Baglie ${ }^{1}$, Me. Mário Popolin Neto ${ }^{2}$, \\ Dra. Marcia Aparecida Zanoli Meira e Silva ${ }^{1}$, Dr. José Remo Ferreira Brega ${ }^{3}$ \\ ${ }^{1}$ Faculdade de Ciências \\ Universidade Estadual Paulista "Júlio de Mesquita Filho" \\ Caixa Postal 14-01 - 17033-360 - Bauru - SP - Brasil \\ ${ }^{2}$ Instituto Federal de São Paulo \\ Caixa Postal 250 - 14801-600 - Araraquara - SP - Brasil \\ ${ }^{3}$ Instituto de Biociências, Letras e Ciências Exatas \\ Universidade Estadual Paulista "Júlio de Mesquita Filho" \\ Caixa Postal 2265 - 15054-000 - São José do Rio Preto - SP - Brasil \\ luizssb.biz@gmail.com, mariopopolin@gmail.com, marciazm@fc.unesp.br, remo@fc.unesp.br
}

\begin{abstract}
O$ aprendizado humano é complexo e requer esforço, porém pode ser facilitado com motivação. Com isso, em conjunto com o avanço da tecnologia voltada a Realidade Virtual (RV), este trabalho apresenta a utilização da $R V$ no ensino científico, particularmente, da química. Adicionalmente, motores de jogo, em especial Unity, tem ganhado popularidade devido a facilidade de desenvolvimento e suporte multi-plataforma. O corrente estudo acompanha a adaptação da aplicação UnityMol, propícia para a visualização $3 D$ de moléculas, para o sistema multi-projetado MiniCAVE, através do componente Unity Cluster Package. A aplicação resultante possibilita a visualização de moléculas em um ambiente imersivo e colaborativo.
\end{abstract}

\section{Introdução}

Levando em conta o processo de aprendizado humano, pode-se ver que ele é consideravelmente complexo, requerendo muito esforço para que seja empregado com frequência e sucesso, o que torna necessária, então, a existência de motivação entre os estudantes, principalmente os mais novos [Virvou et al. 2005].

Um fator que causa o aumento da motivação (e consequente aumento no aprendizado) é a paridade entre o estilo de aprendizado da pessoa e o modo com que ela recebe a instrução [Mikropoulos and Natsis 2011], sendo que o estilo visual-espacial tem certa proeminência entre os estilos de aprendizado naturais [Gardner 1983].

Em conjunto, os avanços das tecnologias voltadas a Realidade Virtual (RV) e Ambientes Virtuais (AVs), tanto em nível de hardware como software, trouxeram a tona maneiras novas e até mesmo antigas (que, agora, se tornaram melhores, viáveis e/ou possíveis) para se interagir com e utilizar da virtualidade nos domínios do conhecimento e da vida do ser humano, além do entretenimento. Alguns autores (e.g. Steuer [Steuer 1992]) definem o uso de RV envolvendo HMDs (head-mounted displays), no entanto, é possível obter o mesmo efeito de imersão através do uso de projetores em sistemas multiprojetados, entre os quais o mais famoso é o CAVE [Cruz-Neira et al. 1992], em que o 
usuário é posto entre quatro "paredes" nas quais são projetadas partes contínuas do AV, fornecendo, assim, a sensação de imersão.

Desde a introdução da capacidade de criação de AVs em RV, duas de suas aplicações mais importantes são aprendizado e treinamento [Casu et al. 2015]. Um dos casos de aprendizado envolve a simulação de ambientes impossíveis de serem sentidos diretamente, como, por exemplo, modelagem química, onde os modelos 3D e técnicas de RV auxiliam na aquisição de conhecimento relativo aos modelos [Dias et al. 2011].

Aplicações de RV podem ser desenvolvidas utilizando diversas ferramentas, entre as quais estão motores de jogo, que fornecem a infraestrutura para criação de jogos (estes, frequentemente, envolvendo $\mathrm{AVs}$ ), agindo como conexão entre lógica própria do jogo e componentes reutilizáveis [Anderson et al. 2013]. Eles tem sido repetidamente usados na ciência devido a relativa baixa curva de aprendizado, a facilidade de desenvolvimento de aplicações com gráficos 3D realistas [Juarez et al. 2009], facilidade de integração com hardware comercial (como HMDs) [Google 2016], entre outras facilidades.

Entre os motores de jogo gratuitos disponíveis no mercado, está o Unity ${ }^{1}$, um motor de jogo completo, com motor de renderização customizado, ferramenta com workflows intuitivos e suporte multi-plataforma [Unity Technologies 2016]. Outra grande vantagem do Unity está na comunidade, que, somente pelos meios oficiais ${ }^{2}$, já disponibilizou mais de 31.000 componentes extras. Assim, o Unity foi escolhido como um das bases para o corrente estudo.

\subsection{Objetivos}

Tendo em vista o emprego de tecnologia de RV na visualização científica (de moléculas), o objetivo principal do estudo é o aprimoramento das maneiras como educadores/profissionais de áreas voltadas a química podem visualizar e apresentar a seus alunos e parceiros estruturas moleculares, bem como aumentar a colaboração entre eles ao tratar do assunto, de maneira a diminuir as barreiras na compreensão.

Para tanto, foi desenvolvida uma aplicação, o Visualizador Imersivo e Colaborativo de Moléculas, utilizando, em suma, um aparato de hardware para exibição (o MiniCAVE [Dias et al. 2012], que fornece a imersão e aspectos colaborativos) e dois aparatos de software (Unity Cluster Package [Neto et al. 2015] e UnityMol [Doutreligne et al. 2014], que fornecem a base de software para a aplicação).

\subsection{Estrutura do Estudo}

A fim de oferecer melhor contextualização a respeito da questão em estudo, na Seção 2 Estudos Correlatos, serão apresentados outros estudos que já abordaram os os temas de visualização imersiva de moléculas e utilização de aplicações de visualização no ensino de química.

Nas Seções 3 MiniCAVE, 4 Unity Cluster Package e 5 UnityMol serão apresentados os principais aparatos de hardware e software sobre os quais o Visualizador Imersivo e Colaborativo de Moléculas foi desenvolvido.

A Seção 6 Visualizador Imersivo e Colaborativo de Moléculas apresenta, então, os detalhes de implementação do estudo, enquanto a Seção 7 Discussão apresenta alguns

\footnotetext{
${ }^{1}$ Unity: http://unity3d.com

${ }^{2}$ Unity Asset Store: https://www.assetstore.unity3d.com
} 
pontos interessantes a respeito do trabalho desenvolvido. Para finalizar o tratamento do Visualizador, a Seção 8 Trabalhos Futuros contém algumas das direções para futuros estudos baseados no trabalho aqui desenvolvido.

\section{Estudos Correlatos}

O estudo mais correlato ao corrente é o realizado por Dias et al [Dias et al. 2011], em que é explorada a exibição de estruturas moleculares em sistemas multi-projetados, para maior imersão. Foi desenvolvido uma aplicação em Java (utilizando a biblioteca Java3D), ChemCAVE3D, que permite o carregamento de modelos 3D nos formatos X3D e VRML e interação com as moléculas através do mouse e teclado ou Kinect. Em pré-avaliação por educadores, houve crença de que o sistema possa ser usado como apoio ao ensino de Química.

Vários estudos [Tüysüz 2010, de Jong et al. 2013, Dalgarno et al. 2009, Altun et al. 2009] abordam a questão de laboratórios virtuais para o aprendizado de ciência. Esses laboratórios são partcularmente úteis, pois evitam os riscos, custos e outros problemas geralmente relacionados a utilização e manutenção de laboratórios físicos. Nos laboratórios virtuais, as leis e propriedades das física, química e biologia são simuladas computacionalmente e é dada o aluno a possibilidade de interagir com objetos, partículas, instrumentos, e assim por diante, a fim de que recrie experimentos que seriam realizados em laboratórios físicos. Outra vantagem, particularmente importante, é a possibilidade de experimentos que seriam de outra forma impossíveis, devido a escala, velocidade, complexidade, etc.

Anderson e Weng [Anderson and Weng 1999] desenvolveram o VRDD (Applying Virtual Reality Visualization To Protein Docking and Design), uma aplicação de visualização em RV do acoplamento e design de proteínas, onde a imagem é projetada na ImmersaDesk, um display semi-imersivo no formato de mesa de rascunho. $\mathrm{O}$ usuário pode visualizar e manipular duas proteínas ao mesmo tempo, mudando detalhes de suas representações e determinando como elas podem se acoplar. Ao fim do estudo, a contribuição encontrada se centrava no fato de a RV aumenta o engajamento dos sentidos visual e motor do usuário num ambiente intuitivo quanto a interação. Outro ponto importante determinado é o de evitar que o usuário se perca na imersão proporcionada pela RV.

No estudo de Drouhard et al. [Drouhard et al. 2015], é utilizado o HMD Oculus Rift para visualização imersiva e exploratória de estruturas de cristais conjuntos de dados de espalhamento de nêutrons. O objetivo era a diminuição das barreiras de entrada para análise exploratória de dados científicos complexos; para tanto, foi desenvolvida uma aplicação no motor de jogos Unity, devido a seu ambiente de desenvolvimento simplificado e facilidades para criação de AVs. Ao fim, os autores reconhecem o potencial do trabalho realizado e também notam que visualizações colaborativas com displays de RV (vários HMDs ou um sistema multi-projetado) são particularmente desejadas para instalações especiais.

\section{MiniCAVE}

O MiniCAVE é um sistema de multi-projeção baseado no CAVE, proposto por [Dias et al. 2012] como uma alternativa simples e de baixo custo. 
V Congresso Brasileiro de Informática na Educação (CBIE 2016)

Anais dos Workshops do V Congresso Brasileiro de Informática na Educação (CBIE 2016)

O MiniCAVE é composto por três telas (de dimensões $2.5 \mathrm{~m}$ x $1.5 \mathrm{~m}$, cada) dispostas num ângulo de $30^{\circ}$ entre si. A cada tela são designados dois projetores BenQ W1000 HD com lentes polarizadas, a fim de oferecer estereoscopia passiva. Cada projetor está conectado a um PC Windows 7 dedicado (Intel Core i7, 8GB RAM e NVIDIA FX 1800 768MB). Todos os PCs se conectam entre si através de um switch Gigabit 3Com.

A Figura 1 apresenta a organização do MiniCAVE. Nela, percebe-se que a disposição das telas tem o propósito de envolver o(s) usuário(s).

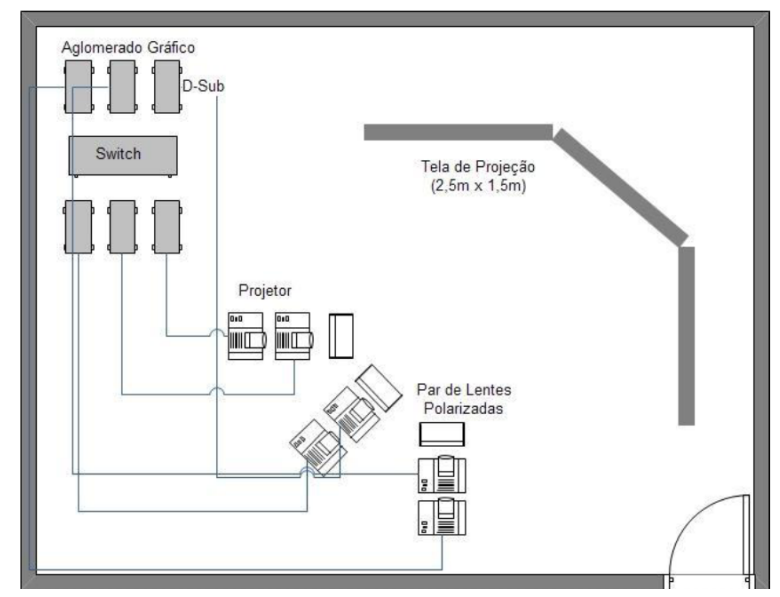

Figura 1. Diagrama da estrutura do MiniCAVE. Fonte: [Dias et al. 2011].

\section{Unity Cluster Package}

O Unity Cluster Package é um pacote para o motor de jogo Unity voltado a facilitar o desenvolvimento de aplicações de RV que utilizam clusters gráficos (conjunto de computadores conectados entre si e realizando uma mesma tarefa compartilhada) através de componentes drag-and-drop [Neto et al. 2015].

Ele é formado pelos seguintes componentes principais [Neto et al. 2015]:

- Multi Projection Camera: consiste numa modificação do componente de câmera original fornecido dentro do Unity, que é responsável por obter a imagem que será mostrada pela aplicação para o usuário. Ele sobreescreve algumas funções da câmera original a fim de que apresente a imagem com distorção adequada quando apontada para um local indicado no AV e forneça efeito de paralaxe (seguindo a cabeça de um usuário específico).

- Node Manager: faz uso do componente de rede nativo do Unity para implementar o modelo de renderização em clusters gráficos mestre-escravo. Ele estabelece a necessidade de classificação dos dispositivos conectados ao cluster em mestre, que é responsável por inicializar a aplicação e, de maneira geral, a coordenar, e escravos, que seguem as ações do mestre, sem desencadear ações próprias. Este componente é responsável por instanciar o Multi Projection Camera em cada computador do cluster e o configurar (seguindo um arquivo de especificação) de maneira que cada computador tenha sua a câmera do visualização do AV construído apontando para um ponto específico.

- Device Manager: permite coleta dos dados de entrada fornecidos por aparelhos de interação, incluindo mouse, teclado joystick e dispositivos móveis. 
V Congresso Brasileiro de Informática na Educação (CBIE 2016)

Anais dos Workshops do V Congresso Brasileiro de Informática na Educação (CBIE 2016)

\section{UnityMol}

UnityMol $^{3}$ é um software código-aberto ${ }^{4}$ que permite a visualização de estruturas moleculares e redes biológicas, seguindo várias metáforas de visualização (e.g. bola-e-vareta, alcaçuz, Van der Waals) [Doutreligne et al. 2014]. Como o nome indica, o UnityMol foi desenvolvido utilizando o motor de jogo Unity.

O UnityMol pode representar moléculas através de texturas e partículas, mas o meio principal utilizado é a representação hyperball, proposta por [Chavent et al. 2011], que foi implementada pelos autores a fim de ser possível representar esferas, varetas e hiperboloides através de um algoritmo unificado.

Moléculas podem ser carregadas a partir de arquivos com formato .PDB ou obtidas pela internet, através de uma pesquisa por ID no Worldwide Protein Data Bank ${ }^{5}$. Uma vez que a molécula esteja carregada, o usuário pode manipulá-la através de giro e zoom, bem como alterar propriedades de sua representação, como escala, cores, texturas, brilho, exibição/oclusão de átomos de hidrogêneo e outros [Doutreligne et al. 2014]. Recursos mais avançados, tais como animações, também são possíveis, embora sejam mais envolvidos no funcionamento.

A Figura 2 apresenta duas possíveis representações de uma mesma molécula pelo UnityMol. É possível perceber que a gama de opções é particularmente compreensiva.

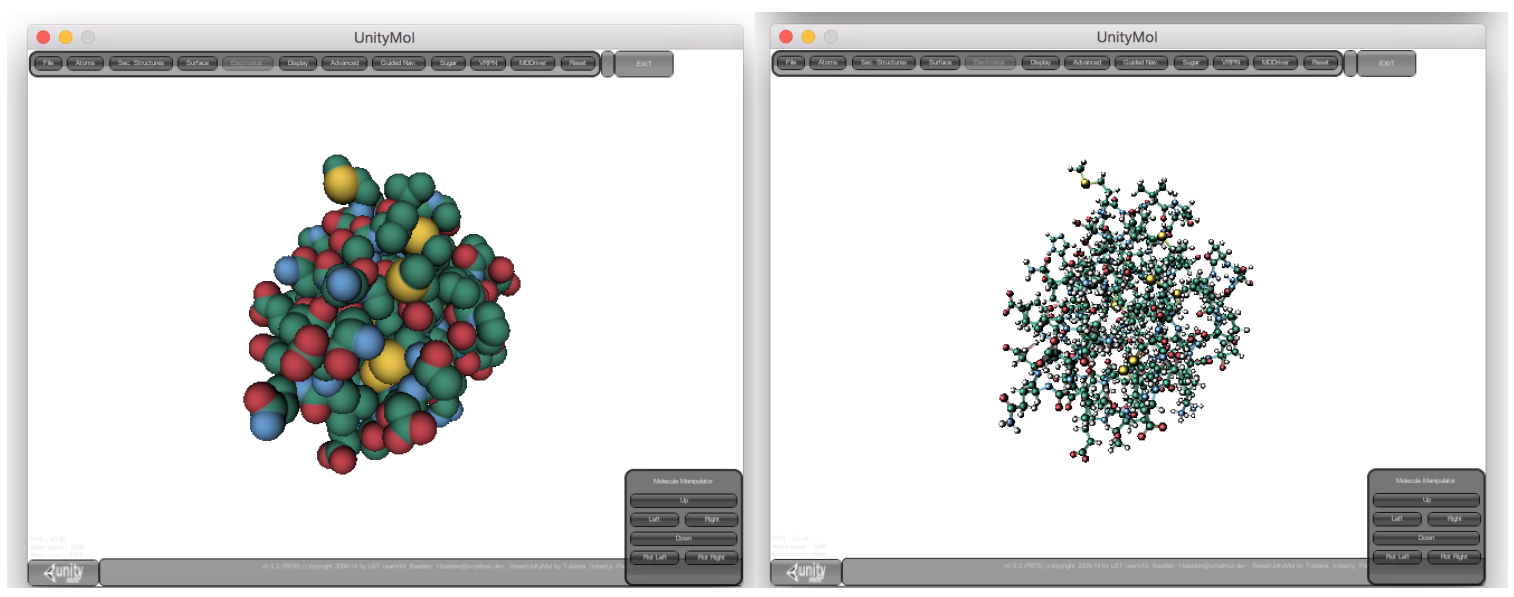

Figura 2. Duas representações da mesma molécula no UnityMol.

\section{Visualizador Imersivo e Colaborativo de Moléculas}

O Visualizador Imersivo e Colaborativo de Moléculas desenvolvido pode ser descrito como um port do UnityMol para sistemas multi-projetados (em particular, o MiniCAVE).

A aplicação final é executada no cluster gráfico do MiniCAVE, utilizando somente três dos projetores disponíveis (um de cada tela), pois não foi empregada a estereoscopia passiva permitida pelo aparato. Os PCs ligados aos projetores utilizados tomaram o papel de escravos do cluster, sendo que foi utilizado como mestre um sétimo PC conectado a

\footnotetext{
${ }^{3}$ UnityMol: http://www.baaden.ibpc.fr/umol/

${ }^{4}$ Código-fonte UnityMol: https://sourceforge.net/projects/unitymol/?source=navbar

${ }^{5}$ Worldwide Protein Data Bank: http://www.wwpdb.org
} 
V Congresso Brasileiro de Informática na Educação (CBIE 2016)

Anais dos Workshops do V Congresso Brasileiro de Informática na Educação (CBIE 2016)

rede, mas independente do cluster do MiniCAVE, utilizando seu próprio monitor separado.

No Visualizador, os menus e controles do UnityMol são exibidos somente no nó mestre, assim, como esse nó exibe seu conteúdo num monitor separado das telas principais do MiniCAVE, é possível que ele seja utilizado como uma Estação de Controle, enquanto as telas do MiniCAVE exibem pura e simplesmente a molécula sendo estudada.

O Visualizador Imersivo e Colaborativo de Moléculas foi posto em execução no Laboratório de Interfaces e Visualização (LIV), da Faculdade de Ciências, dentro da Unesp - Bauru, onde está montado o MiniCAVE. Um esquema da disposição dos elementos no LIV é apresentado na Figura 3. Na Figura, a região dentro do contorno vermelho indica onde estão as carteiras nas quais alunos e/ou colegas de trabalho podem observar as moléculas. Também é possível ver que a Estação de Controle, na forma de um computador verde, situa-se ao lado do MiniCAVE; o conteúdo exibido na Estação de Controle equivale ao conteúdo exibido na tela central do MiniCAVE e isso acontece pois os Multi Projection Cameras desses nós estão configurados para apontar para a exata mesma parte do AV da molécula, assim, o operador da Estação de Controle tem a melhor visão (dentro das limitações de espaço das telas e monitor) do contexto geral do AV onde está a molécula.

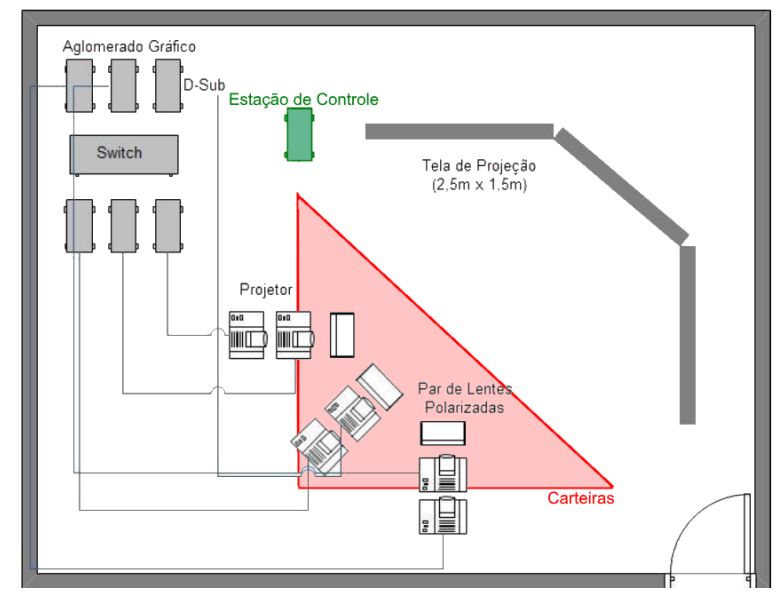

Figura 3. Disposição dos elementos do Visualizador Imersivo e Colaborativo de Moléculas.

\section{Discussão}

O corrente estudo visou a adaptação de um software código-aberto de visualização científica de estruturas moleculares para um sistema multi-projetado.

A aplicação resultante permite que moléculas sejam exibidas no que essencialmente corresponde a uma área contínua de 7.5 metros de largura por 1.5 metro de altura (área de 11.25 metros quadrados), disposta de maneira angular, de modo a envolver os usuários, proporcionando imersão no $\mathrm{AV}$ em que se situa a molécula em estudo. $\mathrm{O}$ fato de que o conteúdo da aplicação é exibido em um conjunto de telas acessível a toda plateia também permite que haja colaboração entre eles, de maneira a promover a discussão e observação do conteúdo por todos de maneira síncrona.

Recursos de customização da molécula oferecidos pela aplicação permitem que as 
V Congresso Brasileiro de Informática na Educação (CBIE 2016)

Anais dos Workshops do V Congresso Brasileiro de Informática na Educação (CBIE 2016)

visualizações sejam efetivas para a plateia ou reforcem certos aspectos determinados pelo operador da estação de controle.

Isso pode ser melhor apreciado nas Figuras 4, 5 e 6, fotos panorâmicas do Visualizador Imersivo e Colaborativo de Moléculas em execução, cada uma exibindo uma possível representação para uma estrutura cristalina de DNA ${ }^{6}$, proposta por [Drew et al. 1981]. Na Figura 4, foi utilizada a representação "hyperball", comentada na Seção 5 UnityMol, sendo que as ligações entre cada átomo são representadas por cubóides. Na Figura 5, foi utilizada a representação "bola-e-vareta", que pode ser melhor para visualizar sítios ativos ou de ligação de proteínas [Kraulis 1991]. Na Figura 6, foi utilizada a representação "alcaçuz" (a qual tem certa semelhança com o doce de mesmo nome) junto a uma textura adicional nos átomos, para efeitos visuais mais interessantes. Essas são só algumas das possíveis maneiras de representar as moléculas na aplicação, já que há vários parâmetros como espessura e cor dos átomos e ligações, oclusão de átomos de hidrogêneo ou exceto hidrogêneo, salientar anéis, entre outros.

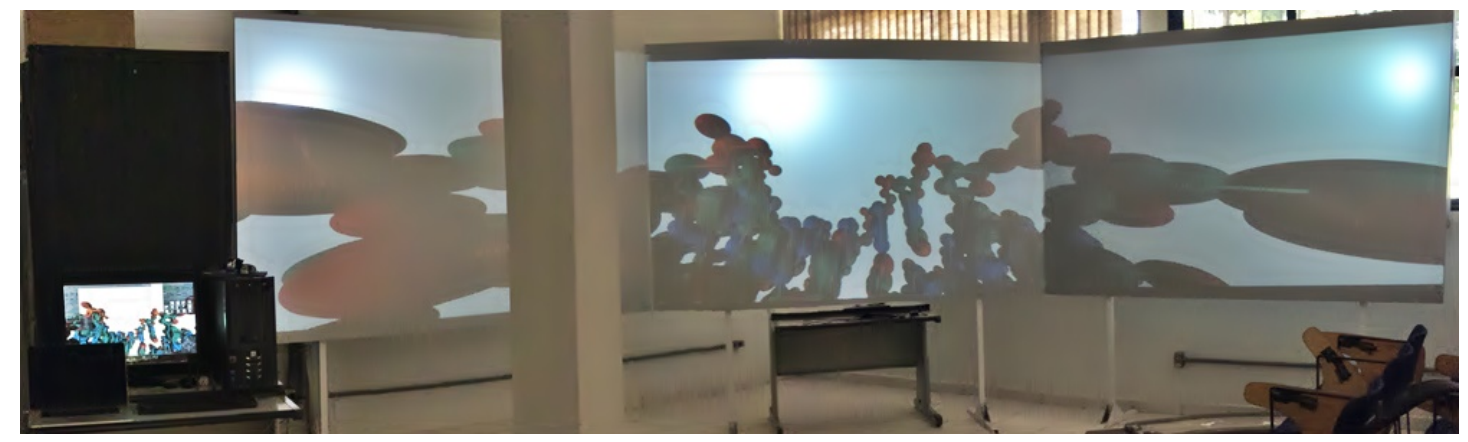

Figura 4. Visualizador Imersivo e Colaborativo de Moléculas com representação "hyperball".

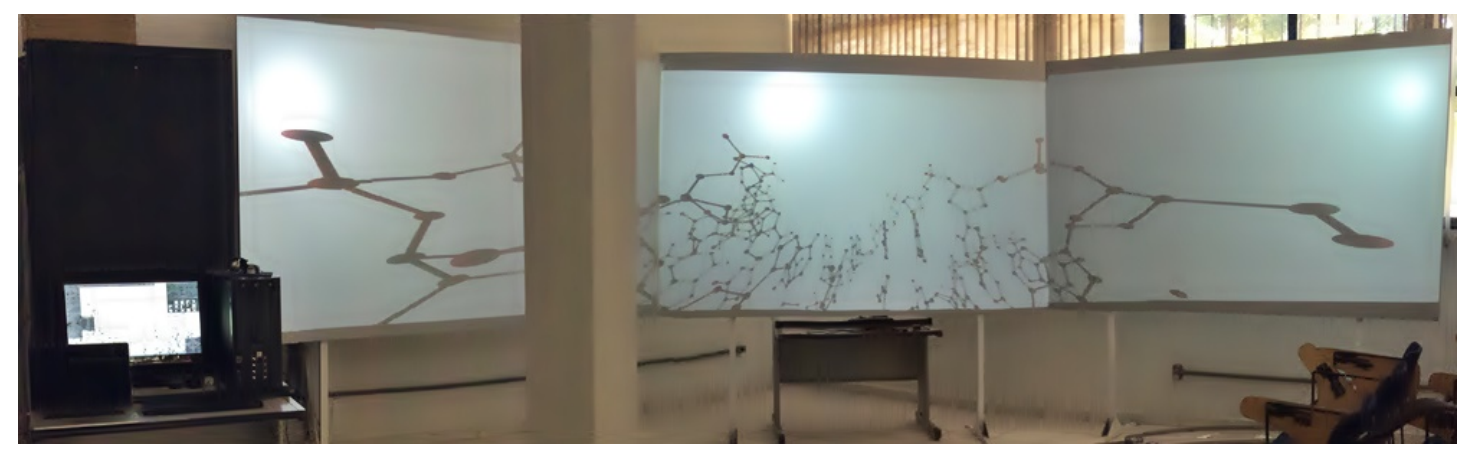

Figura 5. Visualizador Imersivo e Colaborativo de Moléculas com representação "bola-e-vareta".

\section{Trabalhos Futuros}

Dado o potencial de ambos o MiniCAVE e Unity Cluster Package para trabalhar com estereoscopia passiva, o primeiro e mais próximo trabalho futuro envolverá a configuração do Visualizador para que trabalhe com estereoscopia passiva.

A validação com professores e alunos da área química será empregada, para que os benefícios proporcionados pelo Visualizador possam ser melhor mensurados e seus recursos sejam otimizados de acordo.

${ }^{6}$ 1BNA: http://www.rcsb.org/pdb/explore/explore.do?structureId=1 bna 
V Congresso Brasileiro de Informática na Educação (CBIE 2016)

Anais dos Workshops do V Congresso Brasileiro de Informática na Educação (CBIE 2016)

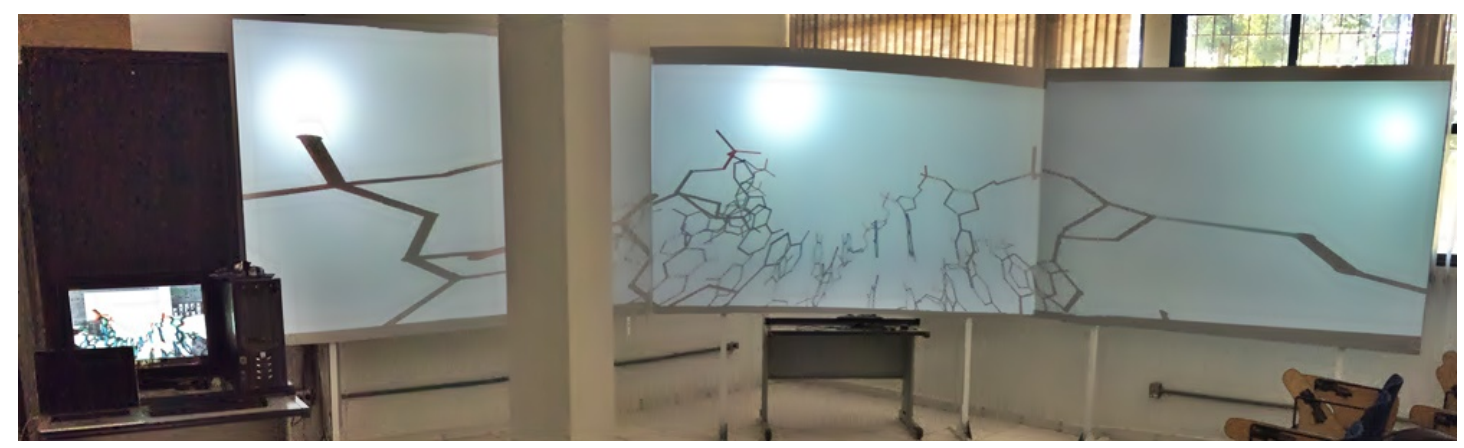

Figura 6. Visualizador Imersivo e Colaborativo de Moléculas com representação "alcaçuz".

Outro item futuro, particularmente importante, envolverá suporte ao uso de dispositivos móveis escravos, para que sejam utilizados como HMDs de relativo baixo custo, através de aparatos como Google Cardboard ${ }^{7}$, permitindo que alunos não só acompanhem a estrutura molecular de maneira ainda mais imersiva, mas também apresentem maior engajamento, pois poderão estar montando o próprio material de aprendizado (o HMD, no caso do Google Cardboard).

\section{Reconhecimentos}

A pesquisa foi parcialmente financiada pelo Conselho Nacional de Desenvolvimento Científico e Tecnológico (CNPq), na forma de bolsa de estudos fornecida ao autor Luiz $\mathrm{S}$. S. Baglie.

Agradecimentos a Dr. Marc Baaden (CNRS - França), um dos desenvolvedores por trás do UnityMol, pelo suporte inicial durante o desenvolvimento do software deste estudo, e, também, de maneira geral, a todo time por trás do UnityMol, ao tornar o códigofonte da aplicação aberto e livre.

\section{Referências}

Altun, E., Demirdağ, B., Feyzioğlu, B., Ateş, A., and İlker Çobanoğlu (2009). Developing an interactive virtual chemistry laboratory enriched with constructivist learning activities for secondary schools. Procedia - Social and Behavioral Sciences, 1(1):18951898.

Anderson, A. and Weng, Z. (1999). VRDD: Applying virtual reality visualization to protein docking and design. Journal of Molecular Graphics and Modelling, 17(34):180-186.

Anderson, E. F., McLoughlin, L., Watson, J., Holmes, S., Jones, P., Pallett, H., and Smith, B. (2013). Choosing the infrastructure for entertainment and serious computer games - a whiteroom benchmark for game engine selection. In Games and Virtual Worlds for Serious Applications (VS-GAMES), 2013 5th International Conference on, pages 1-8.

Casu, A., Spano, L. D., Sorrentino, F., and Scateni, R. (2015). RiftArt : Bringing Masterpieces in the Classroom through Immersive Virtual Reality. STAG: Smart Tools \& Apps for Graphics.

\footnotetext{
${ }^{7}$ Google Cardboard: https://vr.google.com/cardboard/get-cardboard/
} 
V Congresso Brasileiro de Informática na Educação (CBIE 2016)

Anais dos Workshops do V Congresso Brasileiro de Informática na Educação (CBIE 2016)

Chavent, M., Vanel, A., Tek, A., Levy, B., Robert, S., Raffin, B., and Baaden, M. (2011). Gpu-accelerated atom and dynamic bond visualization using hyperballs: A unified algorithm for balls, sticks, and hyperboloids. Journal of computational chemistry, 32(13):2924-2935.

Cruz-Neira, C., Sandin, D. J., DeFanti, T. A., Kenyon, R. V., and Hart, J. C. (1992). The cave: Audio visual experience automatic virtual environment. Commun. ACM, 35(6):64-72.

Dalgarno, B., Bishop, A. G., Adlong, W., and Bedgood, D. R. (2009). Effectiveness of a Virtual Laboratory as a preparatory resource for Distance Education chemistry students. Computers and Education, 53(3):853-865.

de Jong, T., Linn, M. C., and Zacharia, Z. C. (2013). Physical and Virtual Laboratories in Science and Engineering Education. Science, 340(6130):305-308.

Dias, D. R. C., Brega, J. R. F., Lamarca, A. F., Neto, M. P., Suguimoto, D. J., Agostinho, I., and Gouveia, A. F. (2011). Chemcave3d: Sistema de visualizaçao imersivo e interativo de moléculas 3d. In WORKSHOP DE REALIDADE VIRTUAL E AUMENTADA, UBERABA-MG.

Dias, D. R. C., Neto, M. P., Brega, J. R. F., Gnecco, B. B., Trevelin, L. C., and de Paiva Guimarães, M. (2012). Design and evaluation of an advanced virtual reality system for visualization of dentistry structures. In Virtual Systems and Multimedia (VSMM), 2012 18th International Conference on, pages 429-435.

Doutreligne, S., Cragnolini, T., Pasquali, S., Derreumaux, P., and Baaden, M. (2014). Unitymol: Interactive scientific visualization for integrative biology. In Large Data Analysis and Visualization (LDAV), 2014 IEEE 4th Symposium on, pages 109-110. IEEE.

Drew, H. R., Wing, R. M., Takano, T., Broka, C., Tanaka, S., Itakura, K., and Dickerson, R. E. (1981). Structure of a b-dna dodecamer: conformation and dynamics. Proceedings of the National Academy of Sciences, 78(4):2179-2183.

Drouhard, M., Steed, C. A., Hahn, S., Proffen, T., Daniel, J., and Matheson, M. (2015). Immersive visualization for materials science data analysis using the oculus rift. In Proceedings of the 2015 IEEE International Conference on Big Data (Big Data), BIG DATA '15, pages 2453-2461, Washington, DC, USA. IEEE Computer Society.

Gardner, H. E. (1983). Frames Of Mind: The Theory Of Multiple Intelligences. Basic Books, 10th edition.

Google (2016). Google VR - Google Developers. Disponível em https://developers.google.com/vr/.

Juarez, A., Schonenberg, W., and Bartneck, C. (2009). Implementing a low-cost CAVE system using the CryEngine2. Entertainment Computing, 1(3-4):157-164.

Kraulis, P. J. (1991). Molscript: a program to produce both detailed and schematic plots of protein structures. Journal of applied crystallography, 24(5):946-950.

Mikropoulos, T. A. and Natsis, A. (2011). Educational virtual environments: A ten-year review of empirical research (1999-2009). Computers \& Education, 56(3):769 - 780. 
V Congresso Brasileiro de Informática na Educação (CBIE 2016)

Anais dos Workshops do V Congresso Brasileiro de Informática na Educação (CBIE 2016)

Neto, M. P., Dias, D. R. C., Trevelin, L. C., de Paiva Guimarães, M., and Brega, J. R. F. (2015). Unity cluster package-dragging and dropping components for multi-projection virtual reality applications based on pc clusters. In International Conference on Computational Science and Its Applications, pages 261-272. Springer.

Steuer, J. (1992). Defining Virtual Reality: Dimensions Determining Telepresence. Journal of Communication, 42(4):73-93.

Tüysüz, C. (2010). The effect of the virtual laboratory on students' achievement and attitude in chemistry. International Online Journal of Educational Sciences, 2(1):3753.

Unity Technologies (2016). Unity - Editor. Disponível em https://unity3d.com/unity/editor.

Virvou, M., Katsionis, G., and Manos, K. (2005). Combining software games with education: evaluation of its educational effectiveness. Educational Technology \& Society, 8(2):54-65. 